\title{
Endocrine disrupting compounds in drinking water supply system and human health risk implication
}

\begin{abstract}
To date, experimental and epidemiological evidence of endocrine disrupting compounds (EDCs) adversely affecting human and animal populations has been widely debated. Notably, human health risk assessment is required for risk mitigation. The lack of human health risk assessment and management may thus unreliably regulate the quality of water resources and efficiency of treatment processes. Therefore, drinking water supply systems (DWSSs) may be still unwarranted in assuring safe access to potable drinking water. Drinking water supply, such as tap water, is an additional and crucial route of human exposure to the health risks associated with EDCs. A holistic system, incorporating continuous research in DWSS monitoring and management using multi-barrier approach, is proposed as a preventive measure to reduce human exposure to the risks associated with EDCs through drinking water consumption. The occurrence of EDCs in DWSSs and corresponding human health risk implications are analyzed using the Needs, Approaches, Benefits, and Challenges (NABC) method. Therefore, this review may act as a supportive tool in protecting human health and environmental quality from EDCs, which is essential for decision-making regarding environmental monitoring and management purposes. Subsequently, the public could have sustainable access to safer and more reliable drinking water.
\end{abstract}

Keyword: Endocrine disrupting compounds (EDCs); Drinking water supply system (DWSS); Drinking water; Human health risk assessment; Human exposure; NABC analysis 